Review article

\title{
Short dental implants versus standard dental implants placed in the posterior jaws: A systematic review and meta-analysis
}

\author{
Cleidiel Aparecido Araujo Lemos ${ }^{\mathrm{a}, *}$, Marcio Luiz Ferro-Alves ${ }^{\mathrm{b}}$, Roberta Okamoto ${ }^{\mathrm{b}, \mathrm{d}}$, \\ Marcos Rogério Mendonça ${ }^{\mathrm{c}}$, Eduardo Piza Pellizzer ${ }^{\mathrm{a}, \mathrm{d}}$ \\ a Department of Dental Materials and Prosthodontics, Araçatuba Dental School, UNESP_Univ Estadual Paulista, Araçatuba, Brazil \\ b Department of Surgery and Integrated Clinics, Araçatuba Dental School, UNESP_Univ Estadual Paulista, Araçatuba, Brazil \\ c Department of Pediatric and Community Dentistry, Araçatuba Dental School, UNESP-Univ Estadual Paulista, Araçatuba, Brazil \\ ${ }^{\mathrm{d}}$ CNPq Fellowship
}

\section{A R T I C L E I N F O}

\section{Article history:}

Received 11 June 2015

Received in revised form 8 January 2016

Accepted 9 January 2016

\section{Keywords:}

Edentulous jaws partially

Marginal bone loss

Prosthesis failures

Complications

Meta-analysis

\begin{abstract}
A B S T R A C T
Objective: The purpose of the present systematic review and meta-analysis was to compare short implants (equal or less than $8 \mathrm{~mm}$ ) versus standard implants (larger than $8 \mathrm{~mm}$ ) placed in posterior regions of maxilla and mandible, evaluating survival rates of implants, marginal bone loss, complications and prosthesis failures.

Data: This review has been registered at PROSPERO under the number CRD42015016588. Main search terms were used in combination: dental implant, short implant, short dental implants, short dental implants posterior, short dental implants maxilla, and short dental implants mandible.

Source: An electronic search for data published up until September/2015 was undertaken using the PubMed/Medline, Embase and The Cochrane Library databases.

Study selection: Eligibility criteria included clinical human studies, randomized controlled trials and/or prospective studies, which evaluated short implants in comparison to standard implants in the same study.

Conclusion: The search identified 1460 references, after inclusion criteria 13 studies were assessed for eligibility. A total of 1269 patients, who had received a total of 2631 dental implants. The results showed that there was no significant difference of implants survival $(P=.24$; RR:1.35; CI: $0.82-2.22)$, marginal bone loss $(P=.06$; MD: -0.20 ; CI: -0.41 to 0.00$)$, complications $(P=.08 ; \mathrm{RR}: 0.54 ; \mathrm{CI}$ : $0.27-1.09)$ and prosthesis failures $(P=.92$; RR:0.96; $\mathrm{CI}$ : 0.44-2.09). Short implants are considered a predictable treatment for posterior jaws. However, short implants with length less than $8 \mathrm{~mm}(4-7 \mathrm{~mm})$ should be used with caution because they present greater risks to failures compared to standard implants.

Clinical significance: Short implants are frequently placed in the posterior area in order to avoid complementary surgical procedures. However, clinicians need to be aware that short implants with length less than $8 \mathrm{~mm}$ present greater risk of failures.
\end{abstract}

() 2016 Published by Elsevier Ltd.

\section{Introduction}

Implants are often used as a treatment option for partially or totally edentulous patients [1]. The success is directly related to the osseointegration process [2], and the use of standard implants allows a larger contact area with the bone tissue, which supports the osseointegration process $[3,4]$. Tooth loss in the posterior jaws favors the resorption process of bone tissue [5], causing greater

\footnotetext{
* Corresponding author at: Department of Dental Materials and Prosthodontics, UNESP_Univ Estadual Paulista, Araçatuba, São Paulo, Brazil. José Bonifácio St, 1193, Araçatuba, São Paulo 16015-050, Brazil. Fax: +55 1836363245.

E-mail address: cleidiel@gmail.com (C.A.A. Lemos).
}

proximity to the inferior alveolar nerve and maxillary sinus, limiting the use of longer implants $[3,6]$.

To overcome these problems, bone grafts or maxillary sinus lifting have been indicated to reestablish the height of restored bone tissue and allow for placement of standard implants. However, these techniques are associated with increased postoperative morbidity, higher costs, and higher risks of complications during patient rehabilitation [5,7]. Thus, short implants are used, which are considered to be simpler and more effective for rehabilitating atrophic ridges later [8].

As there is no consensus about the definition of short implants, with some authors considering them to be $<10 \mathrm{~mm}[9,10]$, while others consider short implants $\leq 8 \mathrm{~mm}[4,11]$. Current clinical 
tendencies consider implants with $7 \mathrm{~mm}$ length or less as short or extra-short implants [12]. The discrepancy in the crown-toimplant ratio can increase the risk of mechanical problems, but it did not increase the risk of peri-implant marginal bone loss [13].

Another important aspect to consider is the implants installation area, because the chances of failure are higher when the implants are installed in low-density bone, such as in the posterior maxilla [14]. However, there is no consensus on the survival rate of short implants in the posterior maxilla and mandible [15]. Some authors have shown low success rates $[6,16]$, while others have found high success rates for short implants [17-19].

The aim of this study was to evaluate the survival rate of short implants (equal or less than $8 \mathrm{~mm}$ ) compared to standard implants (larger than $8 \mathrm{~mm}$ ) in the posterior jaws. The null hypotheses are: (1) there are no differences between short implants and standard implants with regard to survival rates of implants and (2) there are no differences in marginal bone loss, complications, and prosthesis failures between short implants and standard implants.

\section{Materials and methods}

\subsection{Registry protocol}

This systematic review was structured following the PRISMA checklist [20] and in accordance with models proposed in the literature [14,21-23]. Moreover, the methods for this systematic review were registered with PROSPERO (CRD42015016588).

\subsection{Eligibility criteria}

The eligible studies should present the following characteristics: (1) randomized controlled trials, (2) prospective studies, (3) with at least ten patients, (4) studies published within last 10 years, (5) comparisons between short implants and standard implants in the same study, (6) published in english.

The exclusion criteria used were: (1) in vitro studies, (2) animal studies, (3) case series or case reports, (4) retrospective studies, (5) computer simulations, (6) patients or data repeated in other included articles, and (7) studies that showed only short implants without comparison group, (8) studies that considered short implants larger than $8 \mathrm{~mm}$.

The PICO approach (population, intervention, comparison, outcomes) was used to address the question: do short implants have similar survival rates compared to standard implants. In this process, the population was patients rehabilitated with dental implants in the posterior jaws (maxilla and mandible). Intervention was short implants in the posterior jaw, and the comparison was made with patients who received standard implants in posterior jaws. The primary outcome evaluated was the survival rates of implants in the posterior jaws. The marginal bone loss, complications, and prosthesis failures were the secondary outcomes.

\subsection{Search strategy and information sources}

The selection of articles was done individually by two of the authors (C.A.A.L. and M.L.F.A.) being selected studies that evaluated the survival of short implants (equal or less than $8 \mathrm{~mm}$ ) installed in maxilla and mandible posterior compared to standard implants (larger than $8 \mathrm{~mm}$ ). Electronic searches were conducted at the selected databases PubMed/Medline, Embase, and Cochrane Library for articles published until 10 September 2015, according to the eligibility criteria. The keywords used in this study were: "short implant AND dental implant OR short dental implants OR short dental implants posterior OR short dental implants maxilla OR short dental implants mandible".
To complement this review, a search in grey literature and manual search in journals specific area was carried out: Clinical Implant Dentistry and Related Research, Clinical Oral Implants Research, International Journal of Oral and Maxillofacial Implants, International Journal of Oral and Maxillofacial Surgery, Journal of Clinical Periodontology, Journal of Dentistry, Journal of Oral and Maxillofacial Surgery, Journal of Oral Implantology, Journal of Oral Rehabilitation, Journal of Periodontology, Periodontology 2000.

\subsection{Data analysis}

One of the authors (C.A.A.L.) collected relevant information from the articles, and a second author (R.O.) checked all of the collected information. A careful analysis was performed to check for disagreements among authors, and a third author (E.P.P.) settled all of the disagreements between the investigators through discussion until a consensus was obtained.

\subsection{Risk of bias}

Two investigators (C.A.A.L. and M.L.F.A.) assessed the methodological quality of studies according to the Jadad scale [24], which ranges from 0 to 5 , with scores of $\geq 3$ considered high quality. The cochrane collaboration criteria for judging risk of bias was used to assess the quality of the studies included for review.

\subsection{Summary measures}

The meta-analysis was based on the Mantel-Haenzel (MH) and Inverse Variance (IV) methods. Survival rates of implants, complications and prostheses failures were the outcome measures evaluated by risk ratio (RR) and marginal bone loss, the continuous outcome were evaluated by mean difference (MD) and the corresponding 95\% confidence intervals (CI). The RR and MD values were considered significant when $P<0.05$. The software reviewer manager 5 (Cochrane Group) was used for meta-analysis.

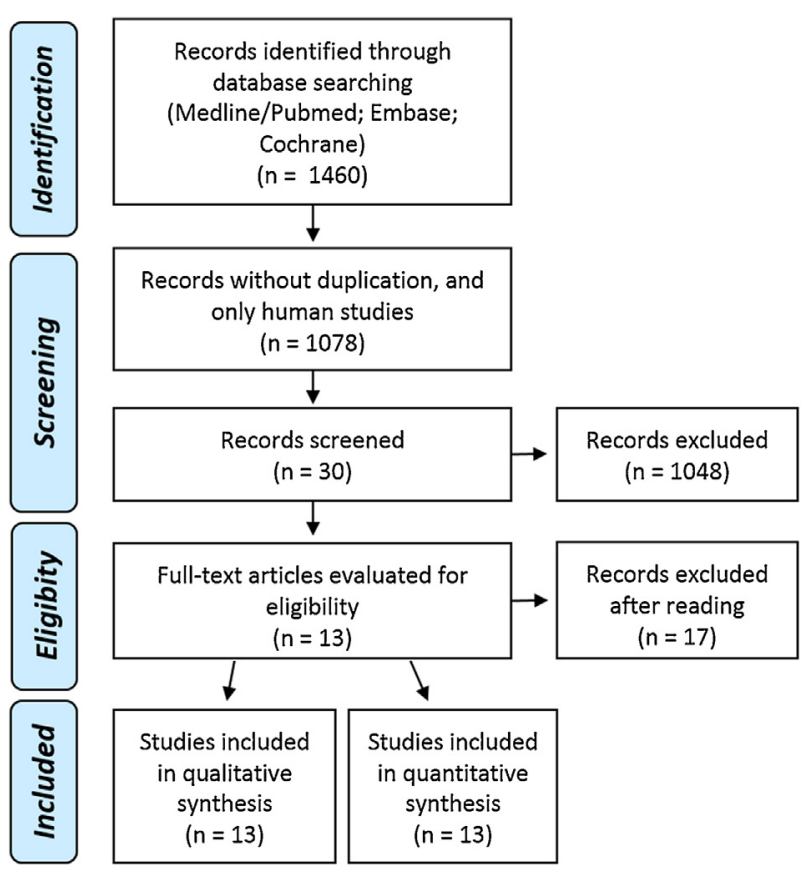

Fig. 1. Flow diagram of the search strategy. 
Table 1

Reasons for the exclusion of 16 articles.

\begin{tabular}{ll}
\hline Reason & References \\
\hline Patients or data repeated in other included articles & {$[37,38,40,41,42,43,44,45]$} \\
Absence of comparison group & {$[46,47,48,50]$} \\
Retrospective study & {$[16,49]$} \\
Not report which group failed & {$[39]$} \\
Considered larger than 8 mm short implants & {$[51,52]$} \\
\hline
\end{tabular}

\subsection{Additional analysis}

The Kappa score was used to calculate the inter-reader agreement during the inclusion process for publication-evaluated databases. Any disagreements were resolved by discussion and consensus of all authors.

\section{Results}

\subsection{Literature search}

The search in the databases retrieved 1460 references, including 1171 from PubMed/MEDLINE, 272 from Embase and 17 from The Cochrane Library. After duplicate references were removed, a detailed review was done on the titles and abstracts of the selected comparative studies, and after applying the inclusion/exclusion criteria, 30 full papers were selected for the eligibility assessment (Fig. 1). After reading the full texts of these articles, 13 studies [6,25-36] were included in the final review. The reasons why studies were excluded [16,37-52] are detailed in Table 1.

\subsection{Description of the studies}

Detailed data of the 13 included studies are listed in Table 2. The 13 selected studies, 10 were RCTs and 3 were prospective, 2631 implants were installed, including 1650 standard implants and 981 short implants installed in 1269 patients with a mean age of 53.43 within the period of January 2004 to 10 September 2015. Ten of the selected studies [25-28,30-34,36] evaluated the survival rates in the maxillary posterior, whereas nine studies $[6,25,26,28-31,33,34]$ evaluated survival rates in the mandibular posterior. One study [35] did not report the region that was rehabilitated.

\subsection{Inter-investigator agreement}

The inter-investigator agreement (Kappa) was calculated by evaluating the selected titles and abstracts, and then obtaining a value for selected articles on PubMed/MEDLINE (kappa $=0.79$ ), Embase (kappa $=0.86$ ) and Cochrane Library (kappa $=1.00)$ presenting a high level of agreement between the reviewers under the Kappa criteria [53].

\subsection{Assessment of study quality}

The Jadad scale was used to check the level of evidence, and indicated that all studies except for three were of high quality (Table 3). The Cochrane criteria indicated low risk of bias for the randomization and allocation of the studies. However, there was high risk of bias concerning the blinding of participants and personnel. This could be justified by the difficulty of blinding the surgeon and patients, especially in the studies that performed bone augmentation procedures. All studies were adequate for addressing incomplete outcome data and were free of selective outcome reporting and other sources of bias (Fig. 2).

\subsection{Survival rates of implants}

The assessed studies [6,25-36] showed that 83 out of 2631 implants placed had failed (3.15\%), comprising 45 standard implants (2.72\%) and 38 short implants (3.87\%) (Fig. 3). A metaanalysis was performed to evaluate whether there was a significant difference due to the increased length of the implants.

A random-effect model found no statistically significant difference between standard implants and short implants placed in the posterior regions ( $P=0.24$; RR: $1.35 ; 95 \% \mathrm{CI}: 0.82-2.22)$ (Fig. 4A). For further analysis, the influence of bone density was evaluated through an analysis of individual jaws. Significant differences for the longer implants were not observed when compared with short implants in the maxilla $(P=0.28$; RR: 1.50 ; 95\% CI: 0.72-3.09), (Fig. 4B) and similarly, no differences were observed in the mandible ( $P=0.34$; RR: 1.52 ; 95\% CI: $0.64-3.63)$ (Fig. 4C).

Due to no consensus about the length of short implants, subanalyses were performed to compare the different lengths $(8 \mathrm{~mm}$ and less than $8 \mathrm{~mm}$ ) individually in relation with standard implants (larger than $8 \mathrm{~mm}$ ). There was no significant difference for $8 \mathrm{~mm}$ implants ( $P=0.34$; RR: $0.50 ; 95 \% \mathrm{CI}: 0.12-2.07)$ (Fig. $5 \mathrm{~A}$ ), but the short implants with length less than $8 \mathrm{~mm}$ showed lower survival rates than standard implants $(P=0.02$; RR: $2.05 ; 95 \% \mathrm{CI}$ : 1.12-3.74) (Fig. 5B).

\subsection{Marginal bone loss}

Nine studies [25,26,28-34] evaluated the differences in length concerning marginal bone loss around the implants through means $(\mathrm{mm})$, which were evaluated by the same studies in different follow-up periods. For the meta-analysis, only the final follow-ups of the studies were used. The overall analysis of studies that evaluated marginal bone loss showed no significant difference between short implants and standard implants $(P=0.06$; MD: -0.20 ; $95 \%$ CI: -0.41 to 0.00 ) (Fig. 6A).

Regarding the rehabilitated arch four studies that did not report the values of marginal bone loss specific to each rehabilitated arch. $[25,26,30,34]$ No differences in marginal bone loss on maxilla were observed [28,31-33] ( $P=0.09$; MD: $-0.19 ; 95 \% \mathrm{CI}:-0.41$ to 0.03$)$ (Fig. 6B), neither were in mandible $[28,29,31,33](P=0.39$; MD: -0.23 ; $95 \%$ CI: -0.76 to 0.30 ) (Fig. $6 \mathrm{C}$ ).

\subsection{Complications rates}

The complication rates were reported by seven studies, which considered any biological or mechanical complications [25,28-33] .Although there were higher rates of complications for the standard implants, there was no significant difference from short implants ( $P=0.08$; RR: 0.54; 95\% CI: 0.27-1.09) (Fig. 7). The mandibular arch had the highest prevalence of biological complication $[28,29,31,33]$. It is noteworthy that most of the studies in this review that reported complication rates performed bone grafting procedures for the installation of standard implants [28-33].

\subsection{Prosthesis failures}

Prosthesis failure rates were evaluated by seven studies [25, 28-33]. The analysis considered prostheses failures that could not be repaired or that failed together with the implant. Thus, no significant differences were observed $(P=0.92$; RR: $0.96 ; 95 \% \mathrm{CI}$ : $0.44-2.09$ ) in relation to the prosthesis failure rates (Fig. 7B). 
Table 2

Characteristics of studies included $(n=13)$.

\begin{tabular}{|c|c|c|c|c|c|c|c|c|c|c|c|}
\hline \multirow{2}{*}{$\begin{array}{l}\text { Author/ } \\
\text { Year }\end{array}$} & \multirow{2}{*}{$\begin{array}{l}\text { Study } \\
\text { Design }\end{array}$} & \multirow{2}{*}{$\begin{array}{l}\text { Length standard } \\
\text { implants and } \\
\text { number of implants } \\
\text { and }\end{array}$} & \multirow{2}{*}{$\begin{array}{l}\text { Length short } \\
\text { implants and } \\
\text { number of } \\
\text { implants }\end{array}$} & \multirow{2}{*}{$\begin{array}{l}\text { Diameter } \\
(ø \mathrm{~mm})\end{array}$} & \multirow{2}{*}{$\begin{array}{l}\text { Implant } \\
\text { systems }\end{array}$} & \multirow{2}{*}{$\begin{array}{l}\text { No. of } \\
\text { patients }\end{array}$} & \multirow{2}{*}{$\begin{array}{l}\text { Mean } \\
\text { age }\end{array}$} & \multirow[t]{2}{*}{ Arch } & \multirow{2}{*}{$\begin{array}{l}\text { Follow- } \\
\text { up }\end{array}$} & \multicolumn{2}{|l|}{ Outcomes measures } \\
\hline & & & & & & & & & & Standard implants & Short implants \\
\hline $\begin{array}{l}\text { Esposito } \\
\text { et al. } \\
\text { [25] }\end{array}$ & $\mathrm{RCT}$ & $\begin{array}{l}\geq 8.5 \mathrm{~mm} \\
69 \text { maxilla } \\
47 \text { mandible }\end{array}$ & $\begin{array}{l}4 \mathrm{~mm} \\
46 \text { maxilla } \\
78 \text { mandible }\end{array}$ & $\begin{array}{l}\text { Standard } \\
4.0 \mathrm{~mm} \\
\text { Short: } \\
4.0 \mathrm{~mm}\end{array}$ & $\begin{array}{l}\text { TwinKon } \\
\text { Universal } \\
\text { SA2-(Global } \\
\text { D) }\end{array}$ & 150 & 55 & $\begin{array}{l}\text { Maxilla } \\
\text { and } \\
\text { Mandible }\end{array}$ & 4 months & $\begin{array}{l}\text { Implants survival: } \\
2 \text { lost } \\
\text { ( } 1 \text { maxilla and } \\
1 \text { mandible) } \\
\text { MBL } \rightarrow 0.43 \\
( \pm 0.25) \\
\text { Prosthesis } \\
\text { survival: } 2 \text { lost } \\
\text { Complications: } 2\end{array}$ & $\begin{array}{l}\text { Implants survival: } \\
3 \text { lost } \\
\text { ( } 1 \text { maxilla and } \\
2 \text { mandible) } \\
\mathrm{MBL} \rightarrow 0.39 \\
( \pm 0.30) \\
\text { Prosthesis } \\
\text { survival: } 2 \text { lost } \\
\text { Complications: } 3\end{array}$ \\
\hline $\begin{array}{l}\text { Rossi et al. } \\
\text { [26] }\end{array}$ & $\mathrm{RCT}$ & $\begin{array}{l}10 \mathrm{~mm} \\
15 \text { maxilla } \\
15 \text { mandible }\end{array}$ & $\begin{array}{l}6 \mathrm{~mm} \\
12 \text { maxilla } \\
18 \text { mandible }\end{array}$ & $\begin{array}{l}\text { Standard } \\
4.1 \mathrm{~mm} \\
\text { Short } \\
4.1 \mathrm{~mm}\end{array}$ & $\begin{array}{l}\text { Straumann } \\
\text { implants }\end{array}$ & 45 & 48.05 & $\begin{array}{l}\text { Maxilla } \\
\text { and } \\
\text { Mandible }\end{array}$ & 5 years & $\begin{array}{l}\text { Implants survival: } \\
1 \text { lost } \\
(1 \text { maxilla }) \\
\text { MBL } \rightarrow 2.64 \\
( \pm 0.56)\end{array}$ & $\begin{array}{l}\text { Implants survival: } \\
4 \text { lost } \\
\text { ( } 3 \text { maxilla and } \\
1 \text { mandible) } \\
\mathrm{MBL} \rightarrow 2.30 \\
( \pm 0.52)\end{array}$ \\
\hline $\begin{array}{c}\text { Thoma } \\
\text { et al. } \\
{[27]}\end{array}$ & $\mathrm{RCT}$ & $\begin{array}{l}11-15 \mathrm{~mm} \\
70 \text { implants }\end{array}$ & $\begin{array}{l}6 \mathrm{~mm} \\
67 \text { implants }\end{array}$ & $\begin{array}{l}\text { Standard } 4 \mathrm{~mm} \\
\text { Short: } \\
4 \mathrm{~mm}\end{array}$ & $\begin{array}{l}\text { OsseoSpeed } \\
4.0 \mathrm{~S} \\
\text { (Astra Tech) }\end{array}$ & 101 & 50.5 & Maxilla & 1 year & $\begin{array}{l}\text { Implants survival: } \\
1 \text { lost }\end{array}$ & $\begin{array}{l}\text { Implants survival: } \\
2 \text { lost }\end{array}$ \\
\hline $\begin{array}{l}\text { Esposito } \\
\text { et al. } \\
\text { [28] }\end{array}$ & $\mathrm{RCT}$ & $\begin{array}{l}\geq 10 \mathrm{~mm} \\
38 \text { maxilla } \\
30 \text { mandible }\end{array}$ & $\begin{array}{l}5 \mathrm{~mm} \\
34 \text { maxilla } \\
26 \text { mandible }\end{array}$ & $\begin{array}{l}\text { Standard } 6 \text { and } \\
4 \mathrm{~mm} \\
\text { Short } \\
6 \mathrm{~mm}\end{array}$ & $\begin{array}{l}\text { Rescue and } \\
\text { EZ Plus } \\
\text { (MegaGen) }\end{array}$ & 30 & 56 & $\begin{array}{l}\text { Maxilla } \\
\text { and } \\
\text { Mandible }\end{array}$ & 3 years & $\begin{array}{l}\text { Implants survival: } \\
2 \text { lost } \\
\text { ( } 1 \text { maxilla and } \\
1 \text { mandible) } \\
\text { Prosthesis } \\
\text { survival: } 0 \text { lost } \\
\text { MBL } \rightarrow \text { Mandible: } \\
1.97 \text { ( } \pm 0.57) \\
\text { Maxilla: } 1.74 \\
\text { ( } \pm 0.57) \\
\text { Complications: } \\
12 \text { mandibular } \\
\text { and } 1 \text { maxillary }\end{array}$ & $\begin{array}{l}\text { Implants survival: } \\
5 \text { lost } \\
\text { ( } 3 \text { maxilla and } \\
2 \text { mandible) } \\
\text { Prosthesis } \\
\text { survival: } 3 \text { lost } \\
\text { MBL } \rightarrow \text { Mandible: } \\
1.79( \pm 0.51) \\
\text { Maxilla: } 1.36 \\
\text { ( } \pm 0.57) \\
\text { Complications: } \\
9 \text { mandibular and } \\
4 \text { maxillary }\end{array}$ \\
\hline $\begin{array}{l}\text { Felice } \\
\text { et al. } \\
\text { [29] }\end{array}$ & $\mathrm{RCT}$ & $\begin{array}{l}\geq 10 \mathrm{~mm} \\
61 \text { implants }\end{array}$ & $\begin{array}{l}6.6 \mathrm{~mm} \\
60 \text { implants }\end{array}$ & $\begin{array}{l}\text { Standard } \\
4 \mathrm{~mm} \\
\text { Short: } \\
4 \mathrm{~mm}\end{array}$ & $\begin{array}{l}\text { Nanotite } \\
\text { parallel } \\
\text { walled } \\
\text { (Biomet 3i) }\end{array}$ & 60 & 55.5 & Mandible & 5 years & $\begin{array}{l}\text { Implants survival: } \\
3 \text { lost } \\
\text { Prosthesis } \\
\text { survival: } 5 \text { lost } \\
\text { MBL } \rightarrow 3.01 \\
( \pm 0.74) \\
\text { Complications: } 25\end{array}$ & $\begin{array}{l}\text { Implants survival: } \\
5 \text { lost } \\
\text { Prosthesis } \\
\text { survival: } 5 \text { lost } \\
\text { MBL } \rightarrow 2.24 \\
( \pm 0.47) \\
\text { Complications: } 6\end{array}$ \\
\hline $\begin{array}{c}\text { Romeo } \\
\text { et al. } \\
{[30]}\end{array}$ & RCT & $\begin{array}{l}10 \mathrm{~mm} \\
19 \text { implants }\end{array}$ & $\begin{array}{l}6 \mathrm{~mm} \\
21 \text { implants }\end{array}$ & $\begin{array}{l}\text { Standard } \\
4 \mathrm{~mm} \\
\text { Short } \\
4 \mathrm{~mm}\end{array}$ & $\begin{array}{l}\text { Straumann } \\
\text { implants }\end{array}$ & 18 & 53 & $\begin{array}{l}\text { Maxilla } \\
\text { and } \\
\text { Mandible }\end{array}$ & 5 years & $\begin{array}{l}\text { Implants survival: } \\
0 \text { lost } \\
\text { Prosthesis } \\
\text { survival: } 0 \text { lost } \\
\text { MBL } \rightarrow 2.99 \\
( \pm 0.90) \\
\text { Complications: } 3\end{array}$ & $\begin{array}{l}\text { Implants survival: } \\
1 \text { lost } \\
\text { Prosthesis } \\
\text { survival: } 1 \text { lost } \\
\text { MBL } \rightarrow 2.97 \\
( \pm 0.47) \\
\text { Complications: } 5\end{array}$ \\
\hline $\begin{array}{l}\text { Queiroz } \\
\text { et al. [6] }\end{array}$ & $\begin{array}{l}\text { Non- } \\
\text { RCT }\end{array}$ & $\begin{array}{l}10 / \\
11.5 \text { mm42 implants }\end{array}$ & $\begin{array}{l}5.5 \text { and } \\
7 \mathrm{~mm} \\
48 \text { implants }\end{array}$ & $\begin{array}{l}\text { Standard } 4 \mathrm{~mm} \\
\text { Short } 5 \mathrm{~mm}\end{array}$ & $\begin{array}{l}\text { Master } \\
\text { Porous } \\
\text { (Conexão) }\end{array}$ & 23 & 53.35 & Mandible & 3 months & $\begin{array}{l}\text { Implants survival: } \\
0 \text { lost }\end{array}$ & $\begin{array}{l}\text { Implants survival: } \\
6 \text { lost }\end{array}$ \\
\hline $\begin{array}{l}\text { Pistili } \\
\text { et al. (A) } \\
\text { [31] }\end{array}$ & $\mathrm{RCT}$ & $\begin{array}{l}\geq 10 \mathrm{~mm} \\
37 \text { maxilla } \\
32 \text { mandible }\end{array}$ & $\begin{array}{l}5 \mathrm{~mm} \\
36 \text { maxilla } \\
31 \text { mandible }\end{array}$ & $\begin{array}{l}\text { Standard } \\
5 \mathrm{~mm} \\
\text { Short } \\
5 \mathrm{~mm}\end{array}$ & $\begin{array}{l}\text { ExFeel } \\
\text { (MegaGen) }\end{array}$ & 80 & 57.75 & $\begin{array}{l}\text { Maxilla } \\
\text { and } \\
\text { Mandible }\end{array}$ & 1 year & $\begin{array}{l}\text { Implant survival: } \\
1 \text { lost } \\
\text { (mandibular) } \\
\text { Failure prosthesis: } \\
2 \text { mandibular } \\
\text { MBL } \rightarrow \text { Mandible: } \\
1.53( \pm 0.29 \text { ) } \\
\text { Maxilla: } 1.09 \\
\text { ( } \pm 0.05) \\
\text { Complications: } \\
17 \text { mandibular } \\
\text { and } 5 \text { maxillary }\end{array}$ & $\begin{array}{l}\text { Implant survival: } \\
1 \text { lost (maxilla) } \\
\text { Failure prosthesis: } \\
1 \text { maxillary } \\
\mathrm{MBL} \rightarrow \text { Mandible: } \\
1.15( \pm 0.12) \\
\text { Maxilla: } 0.87 \\
( \pm 0.07) \\
\text { Complications: } \\
8 \text { mandibular }\end{array}$ \\
\hline $\begin{array}{l}\text { Canizzaro } \\
\text { et al. } \\
{[32]}\end{array}$ & $\mathrm{RCT}$ & $\begin{array}{l}\geq 10 \mathrm{~mm} \\
44 \text { implants }\end{array}$ & $\begin{array}{l}8 \mathrm{~mm} \\
38 \text { implants }\end{array}$ & $\begin{array}{l}\text { Standard } \\
3.7 \text { and } 4.7 \mathrm{~mm} \\
\text { Short } \\
4.7 \text { and } 6 \mathrm{~mm}\end{array}$ & $\begin{array}{l}\text { TS Vent MP- } \\
\text { 1HA Dual } \\
\text { Transition } \\
\text { (Zimmer } \\
\text { Dental) }\end{array}$ & 40 & 50.4 & Maxilla & 5 years & $\begin{array}{l}\text { Implants survival: } \\
5 \text { lost } \\
\text { Prosthesis failure: } \\
2 \\
\text { MBL: } 0.72( \pm 0.41) \\
\text { Complications: } 8\end{array}$ & $\begin{array}{l}\text { Implants survival: } \\
1 \text { lost } \\
\text { Prosthesis Failure: } \\
1 \\
\text { MBL: } 0.41( \pm 0.42) \\
\text { Complications: } 4\end{array}$ \\
\hline $\begin{array}{l}\text { Pistili } \\
\text { et al. (B) } \\
\text { [33] }\end{array}$ & $\mathrm{RCT}$ & $\begin{array}{l}\geq 10 \mathrm{~mm} \\
44 \text { maxilla } \\
47 \text { mandible }\end{array}$ & $\begin{array}{l}6 \mathrm{~mm} \\
39 \text { maxilla } \\
41 \text { mandible }\end{array}$ & $\begin{array}{l}\text { Standard } \\
4 \mathrm{~mm} \\
\text { Short } \\
4 \mathrm{~mm}\end{array}$ & $\begin{array}{l}\text { Southern } \\
\text { Implants }\end{array}$ & 40 & 55.85 & $\begin{array}{l}\text { Maxilla } \\
\text { and } \\
\text { Mandible }\end{array}$ & 1 year & $\begin{array}{l}\text { Implant survival: } \\
3 \text { lost (mandible) } \\
\text { Prosthesis failure: } \\
2 \text { mandibular } \\
\mathrm{MBL} \rightarrow \text { Mandible: } \\
1.03( \pm 0.07)\end{array}$ & $\begin{array}{l}\text { Implant survival: } \\
0 \text { lost } \\
\text { Failure prosthesis: } \\
0 \text { lost } \\
\text { MBL } \rightarrow \text { Mandible: } \\
1.41( \pm 0.31)\end{array}$ \\
\hline
\end{tabular}


Table 2 (Continued)

\begin{tabular}{|c|c|c|c|c|c|c|c|c|c|c|c|}
\hline \multirow{2}{*}{$\begin{array}{l}\text { Author/ } \\
\text { Year }\end{array}$} & \multirow{2}{*}{$\begin{array}{l}\text { Study } \\
\text { Design }\end{array}$} & \multirow{2}{*}{$\begin{array}{l}\text { Length standard } \\
\text { implants and } \\
\text { number of implants } \\
\text { and }\end{array}$} & \multirow{2}{*}{$\begin{array}{l}\text { Length short } \\
\text { implants and } \\
\text { number of } \\
\text { implants }\end{array}$} & \multirow{2}{*}{$\begin{array}{l}\text { Diameter } \\
(ø \mathrm{~mm})\end{array}$} & \multirow{2}{*}{$\begin{array}{l}\text { Implant } \\
\text { systems }\end{array}$} & \multirow{2}{*}{$\begin{array}{l}\text { No. of } \\
\text { patients }\end{array}$} & \multirow{2}{*}{$\begin{array}{l}\text { Mean } \\
\text { age }\end{array}$} & \multirow[t]{2}{*}{ Arch } & \multirow{2}{*}{$\begin{array}{l}\text { Follow- } \\
\text { up }\end{array}$} & \multicolumn{2}{|l|}{ Outcomes measures } \\
\hline & & & & & & & & & & Standard implants & Short implants \\
\hline & & & & & & & & & & $\begin{array}{l}\text { Maxilla: } 0.94 \\
( \pm 0.05) \\
\text { Complications: } \\
10 \text { mandibular e } \\
4 \text { maxillary }\end{array}$ & $\begin{array}{l}\text { Maxilla: } 1.02 \\
( \pm 0.06) \\
\text { Complications: } 0\end{array}$ \\
\hline $\begin{array}{l}\text { Guljé et al. } \\
\text { [34] }\end{array}$ & RCT & $\begin{array}{l}11 \mathrm{~mm} \\
101 \text { implants }\end{array}$ & $\begin{array}{l}6 \mathrm{~mm} \\
107 \text { implants }\end{array}$ & $\begin{array}{l}\text { Standard } \\
4 \mathrm{~mm} \\
\text { Short } \\
4 \mathrm{~mm}\end{array}$ & $\begin{array}{l}\text { OsseoSpeed } \\
4.0 \mathrm{~S} \\
\text { (Astra Tech) }\end{array}$ & 95 & 54.5 & $\begin{array}{l}\text { Maxilla } \\
\text { and } \\
\text { Mandible }\end{array}$ & 1 year & $\begin{array}{l}\text { Implants survival: } \\
1 \text { lost } \\
\mathrm{MBL} \rightarrow 0.02 \\
( \pm 0.60)\end{array}$ & $\begin{array}{l}\text { Implants survival: } \\
3 \text { lost } \\
\mathrm{MBL} \rightarrow 0.06 \\
( \pm 0.27)\end{array}$ \\
\hline Arlin [35] & $\begin{array}{l}\text { Non- } \\
\text { RCT }\end{array}$ & $\begin{array}{l}10-16 \mathrm{~mm} \\
454 \text { implants }\end{array}$ & $\begin{array}{l}6 \text { and } 8 \mathrm{~mm} \\
176 \text { implants }\end{array}$ & $\begin{array}{l}3.3 ; 3.5 ; \\
4.1 \text { and } 4.8 \mathrm{~mm}\end{array}$ & $\begin{array}{l}\text { Straumann } \\
\text { Implants }\end{array}$ & 264 & - & - & 5 years & 12 implants lost & 3 implants lost \\
\hline $\begin{array}{l}\text { Ferrigno } \\
\text { et al. } \\
\text { [36] }\end{array}$ & $\begin{array}{l}\text { Non- } \\
\text { RCT }\end{array}$ & $\begin{array}{l}10 \text { and } 12 \mathrm{~mm} \\
485 \text { implants }\end{array}$ & $\begin{array}{l}8 \mathrm{~mm} \\
103 \text { implants }\end{array}$ & $\begin{array}{l}4.1 \text { and } \\
4.8 \mathrm{~mm}\end{array}$ & $\begin{array}{l}\text { Straumann } \\
\text { Implants }\end{array}$ & 323 & 51.2 & Maxilla & 12 years & 14 implants lost & 4 implants lost \\
\hline
\end{tabular}

$\mathrm{MBL}=$ Marginal bone loss

\section{Discussion}

Recently, some revisions have verified that short implants are a predictable alternative treatment for the edentulous in the posterior region [10,54]. However, the present systematic review only included studies with direct comparisons between short and standard implants, in order to reduce bias.

Short implants are considered an alternative for rehabilitation that reduces the complexity of the treatment, mainly in the posterior jaws. This is due to bone resorption maintaining proximity to anatomical structures, which may compromise standard implant placement [34,54]. There is no consensus in the literature on the performance of short implants compared to standard implants [6], although short implants are usually associated with higher rates of failure due to the reduced contact between the bone and implant compared to longer implants [55], with support primary stability and therefore osseointegration [56]. Furthermore, there is no consensus about the length of an implant for it to be considered short. Thus, sub-analysis was performed with implants of $8 \mathrm{~mm}$ long, and less than $8 \mathrm{~mm}$.

The first hypothesis was partially accepted since there are no differences between short and standard implants about survival rates for the implants in posterior jaws to short implants. These results are supported by recent studies [17-19,57] that found high success rates for short implants. However, when evaluating implants with length less than $8 \mathrm{~mm}$, there were lower survival rates in short implants than standard implants. This is in agreement with studies that reported low success rates for these implants $[16,58]$.

Therefore, $8 \mathrm{~mm}$ implants may be considered standard implants in current clinical tendencies, since the results are similar to those of implants with larger lengths. Short implants with length $<8 \mathrm{~mm}$ should be used with caution in the posterior jaw because the survival rates are reduced significantly when compared to standard implants.

Some factors have contributed to survival rates of implants, such as surface treatment, which may increase the success of short implants compared with machined implants [14,59,60]. The porosity of the surface ensures greater contact between bone and implant. This favors the process of osseointegration $[4,61]$ since minimal surface roughness results in a weak bone-toimplant interface with minimal resistance to tensile forces arising from off-axis loads [6].

Deporter et al. [62] reported that implant surface modification is a key factor in the performance and survival rate of short implants. This may have favored the results of this study, because all of the evaluated studies used implants with surface treatment [6,27-36], although the authors did not report using surface treatment only for short implants.

Although biomechanical studies have reported that the discrepancy of the crown-to-implant ratio may increase the marginal bone loss $[8,63]$, this relation has not been observed

Table 3

Quality assessment of the selected studies.

\begin{tabular}{|c|c|c|c|c|c|c|c|c|c|c|c|c|c|}
\hline \multicolumn{14}{|l|}{ Studies } \\
\hline Quality criteria & $\begin{array}{l}\text { Esposito } \\
\text { et al. } \\
\text { [25] }\end{array}$ & $\begin{array}{l}\text { Rossi } \\
\text { et al. } \\
{[26]}\end{array}$ & $\begin{array}{l}\text { Thoma } \\
\text { et al. } \\
\text { [27] }\end{array}$ & $\begin{array}{l}\text { Esposito } \\
\text { et al. } \\
{[28]}\end{array}$ & $\begin{array}{l}\text { Felice } \\
\text { et al. } \\
{[29]}\end{array}$ & $\begin{array}{l}\text { Romeo } \\
\text { et al. } \\
\text { [30] }\end{array}$ & $\begin{array}{l}\text { Queiroz } \\
\text { et al. [6] }\end{array}$ & $\begin{array}{l}\text { Pistili } \\
\text { et al. (A) } \\
\text { [31] }\end{array}$ & $\begin{array}{l}\text { Cannizzaro } \\
\text { et al. [32] }\end{array}$ & $\begin{array}{l}\text { Pistili } \\
\text { et al. (B) } \\
\text { [33] }\end{array}$ & $\begin{array}{l}\text { Guljé } \\
\text { et al. } \\
{[34]}\end{array}$ & $\begin{array}{l}\text { Arlin } \\
{[35]}\end{array}$ & $\begin{array}{l}\text { Ferrigno } \\
\text { et al. [36] }\end{array}$ \\
\hline $\begin{array}{l}\text { 1. Was the study described as } \\
\text { random? }\end{array}$ & Yes & Yes & Yes & Yes & Yes & Yes & No & Yes & Yes & Yes & Yes & No & No \\
\hline $\begin{array}{l}\text { 2. Was the randomization } \\
\text { scheme described and } \\
\text { appropriate? }\end{array}$ & Yes & Yes & Yes & Yes & Yes & Yes & No & Yes & Yes & Yes & Yes & No & No \\
\hline $\begin{array}{l}\text { 3. Was the study described as } \\
\text { double-blind? }\end{array}$ & No & No & No & No & No & No & No & No & No & No & No & No & No \\
\hline $\begin{array}{l}\text { 4. Was the method of double } \\
\text { blinding appropriate? }\end{array}$ & No & No & No & No & No & No & No & No & No & No & No & No & No \\
\hline $\begin{array}{l}\text { 5. Was there a description of } \\
\text { dropouts and withdrawals? }\end{array}$ & Yes & Yes & Yes & Yes & Yes & Yes & Yes & Yes & Yes & Yes & Yes & Yes & Yes \\
\hline Jadad score & 3 & 3 & 3 & 3 & 3 & 3 & 1 & 3 & 3 & 3 & 3 & 1 & 1 \\
\hline Quality of study & High & High & High & High & High & High & Low & High & High & High & High & Low & Low \\
\hline
\end{tabular}




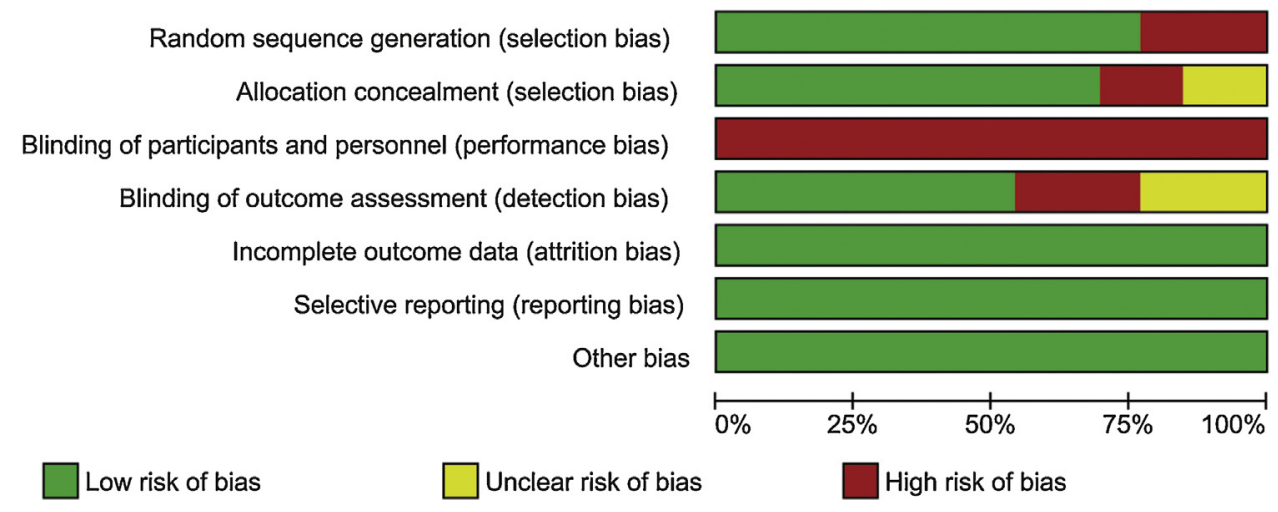

Fig. 2. Assessing risk of bias in included studies by Cochrane risk of bias tools.

through clinical studies $[13,64]$, thereby confirming the results of this study, since short implants did not influence marginal bone loss when compared to standard implants. However, this factor was considered as a limitation because the crown-to-implant ratio was reported by only one study [26].

The type of prosthetic configuration of the implants was considered a limitation of this study. Three studies [26,27,34] reported the use of non-splinted crowns, most of studies performed splinting of crowns for both the short implant and standard implant groups. The authors did not report the influence of splinting as a factor analysis. This may have influenced the results, since splinting short implants is more favorable for reducing stress, and it allows the stress to be shared between the implants $[16,65]$. Splinting factor should be considered for future clinical studies on mainly the posterior jaws as it can undermine the success of short implants if the implants are not splinted [15].

Some studies have suggested that implant diameter influences survival rates of the implants [66,67], so the use of wide-diameter $(\geq 5 \mathrm{~mm})$ in short implants could be favorable. However, studies that used short implants with wide diameter in this review did not show a significant influence $[6,28,31]$. This is supported by another study that reported the role of diameter as a secondary factor in the long-term survival of dental implants placed in the posterior atrophic maxilla [68].

The second hypothesis was accepted, since no significant differences were found in marginal bone loss, complication rates, and prosthesis failures between short implants and standard implants. As mentioned the splinting of crowns can influence to maintaining of marginal bone tissue. Another important factor is the distribution of occlusal contacts made by the prosthesis, since the absence of a masticatory overload does not compromise the peri-implant bone tissue, and values say within the limits established in the literature [69].

Although there were high complication rates (biological or mechanical) for standard implants, there was no significant difference. It should be noted that the large number of complications for standard implants is directly influenced by complementary surgical procedures with implant placement $[28,29,31-33]$, such as maxillary sinus lifting and block bone grafting to the lower jaw. These procedures are related to greater post-operative complications, such as paresthesia, sinus membrane perforation, and infection of the grafted area [28,31-33].

Complementary surgical procedures for bone gain may compromise the patient's acceptance, as well as increase the risk of biological complications, morbidity, costs and, surgical time [70,71], resulting

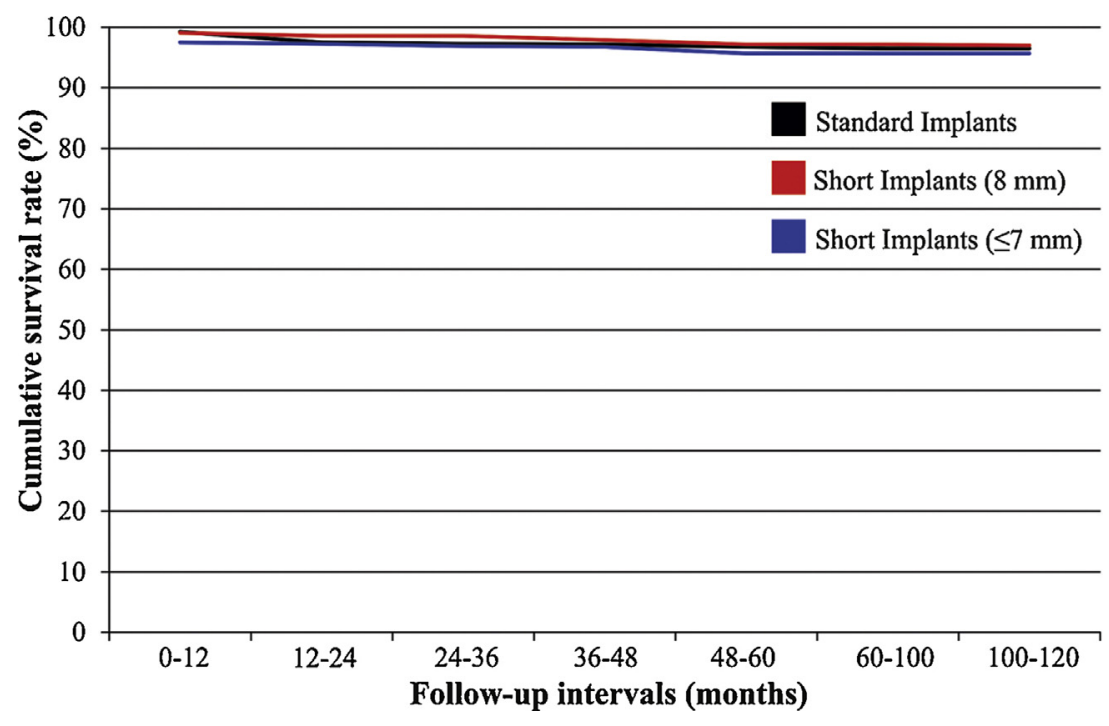

Fig. 3. Kaplan-Meier of cumulative survival rate (\%) of short and standard implants. 


\begin{tabular}{|c|c|c|c|c|c|}
\hline \multirow{2}{*}{$\begin{array}{l}\text { A } \\
\text { Study or Subgroup }\end{array}$} & \multicolumn{2}{|c|}{ Short Implants } & \multicolumn{2}{|c|}{ Standard Implants } & \multirow[b]{2}{*}{ Weight } \\
\hline & Events & Total & Events & Total & \\
\hline Arlin, 2006 & 3 & 176 & 12 & 454 & $15.9 \%$ \\
\hline Canizzaro et al. 2013 & 1 & 44 & 5 & 38 & $5.7 \%$ \\
\hline Esposito et al. 2014 & 5 & 60 & 2 & 68 & $9.7 \%$ \\
\hline Esposito et al. 2015 & 3 & 124 & 2 & 116 & $8.0 \%$ \\
\hline Felice et al. 2014 & 5 & 60 & 3 & 61 & $13.0 \%$ \\
\hline Ferrigno et al. 2006 & 4 & 103 & 14 & 485 & $21.0 \%$ \\
\hline Guljé et al. 2012 & 3 & 107 & 1 & 101 & $5.0 \%$ \\
\hline Pistili et al. $2013(\mathrm{~A})$ & 1 & 68 & 1 & 68 & $3.3 \%$ \\
\hline Pistili et al. 2013 (B) & 0 & 80 & 3 & 91 & $2.9 \%$ \\
\hline Queiroz et al. 2014 & 6 & 48 & 0 & 42 & $3.1 \%$ \\
\hline Romeo et al. 2014 & 1 & 21 & 0 & 19 & $2.5 \%$ \\
\hline Rossi et al. 2015 & 4 & 30 & 1 & 30 & $5.5 \%$ \\
\hline Thoma et al. 2014 & 2 & 67 & 1 & 70 & $4.4 \%$ \\
\hline Total $(95 \% \mathrm{Cl})$ & & 988 & & 1643 & $100.0 \%$ \\
\hline Total events & 38 & & 45 & & \\
\hline
\end{tabular}

Risk Ratio Risk Ratio

M-H, Random, 95\% Cl M-H, Random, $95 \% \mathrm{Cl}$

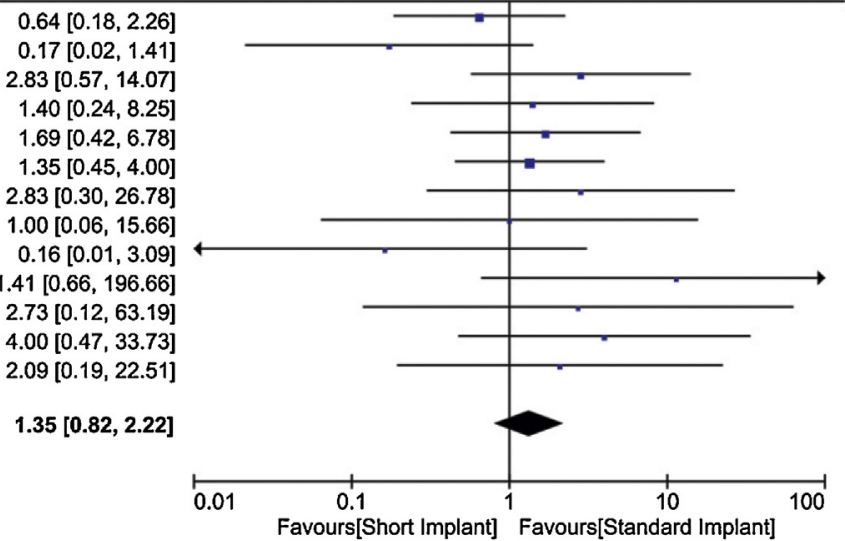

B

Short Implants Standard Implants

Risk Ratio Study or Subgroup Events Total Events Total Weight M-H, Random, 95\% Cl $0.23[0.03,1.90]$ $3.35[0.37,30.73]$ $1.66[0.11,25.91]$ $1.35[0.45,4.00]$ Esposito et al. 2014 $\begin{array}{llll}38 & 5 & 44 & 11.9 \%\end{array}$ Esposito et al. 2015 Ferrigno et al. 2006 Pistili et al. 2013 (A) Pistili et al. 2013 (B) Rossi et al. 2015 Thoma et al. 2014

Total $(95 \% \mathrm{Cl})$ $\begin{array}{llll}34 & 1 & 38 & 10.7 \%\end{array}$ $\begin{array}{llll}47 & 1 & 78 & 7.0 \%\end{array}$ $\begin{array}{llll}103 & 14 & 485 & 44.3 \%\end{array}$ $\begin{array}{llll}36 & 0 & 37 & 5.2 \%\end{array}$ $39 \quad 0 \quad 44$ $\begin{array}{llll}32 & 1 & 15 & 11.6 \%\end{array}$ $3.08[0.13,73.24]$ Not estimable $3.75[0.44,31.62]$ $2.09[0.19,22.51]$ Heterogeneity: $\mathrm{Tau}^{2}=0.00 ; \mathrm{Chi}^{2}=4.60, \mathrm{df}=6(\mathrm{P}=0.60) ;\left.\right|^{2}=0 \%$ Test for overall effect: $Z=1.09(P=0.28)$

$1.50[0.72,3.09]$

\begin{tabular}{|c|c|c|c|c|c|}
\hline \multirow{2}{*}{ Study or Subgroup } & \multicolumn{2}{|c|}{ Short Implants } & \multicolumn{2}{|c|}{ Standard Implants } & \multirow[b]{2}{*}{ Weight } \\
\hline & Events & Total & Events & Total & \\
\hline Esposito et al. 2014 & 2 & 26 & 1 & 30 & $13.8 \%$ \\
\hline Esposito et al. 2015 & 2 & 69 & 1 & 46 & $13.5 \%$ \\
\hline Felice et al. 2014 & 5 & 60 & 3 & 61 & $39.4 \%$ \\
\hline Pistili et al. 2013 (A) & 0 & 31 & 1 & 32 & $7.6 \%$ \\
\hline Pistili et al. 2013 (B) & 0 & 41 & 3 & 47 & $8.8 \%$ \\
\hline Queiroz et al. 2014 & 6 & 48 & 0 & 42 & $9.3 \%$ \\
\hline Rossi et al. 2015 & 1 & 18 & 0 & 15 & $7.7 \%$ \\
\hline Total $(95 \% \mathrm{Cl})$ & & 293 & & 273 & $100.0 \%$ \\
\hline Total events & 16 & & 9 & & \\
\hline Heterogeneity: Tau ${ }^{2}$ & $\mathrm{Chi}^{2}$ & & 0.5 & & \\
\hline
\end{tabular}

Risk Ratio

M-H, Random, 95\% Cl $2.31[0.22,24.01]$

$1.33[0.12,14.28]$ $1.69[0.42,6.78]$

$0.34[0.01,8.13]$

$0.16[0.01,3.07]$ $11.41[0.66,196.66]$ $2.53[0.11,57.83]$ $1.52[0.64,3.63]$
Risk Ratio M-H, Random, 95\% Cl

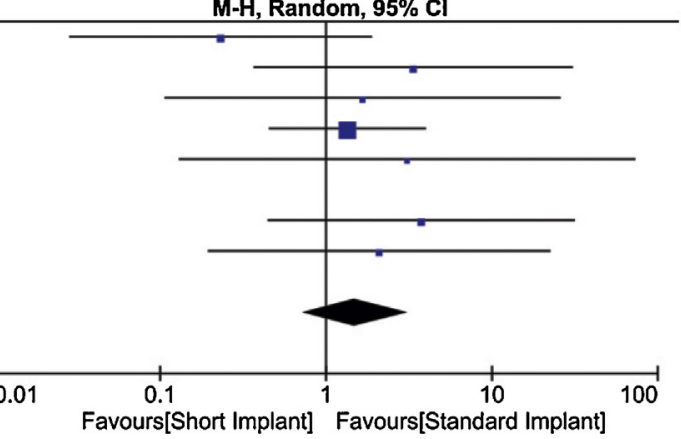

Risk Ratio M-H, Random, 95\% Cl

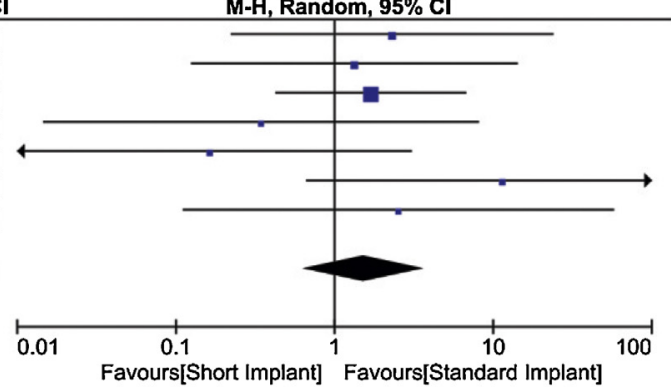

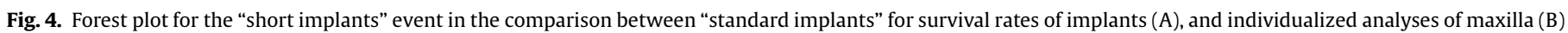
and mandible (C).

in long treatment periods [5]. Thus, short dental implants may represent a favorable treatment alternative [27], corroborating with recent reviews [70,71], which report that it is preferable to use short implants rather complementary surgical procedures. However, in this study, we found that short implants $<8 \mathrm{~mm}(4-7 \mathrm{~mm})$ showed greater risk of implant failure than standard implants. Thus, we propose the use of an implant of appropriate length based on the vertical height of the available bone.

Prosthesis failures were most often associated with failure of the implants [31,33]. However, some studies was necessary to replace of the prostheses due to aesthetic reasons or fractures of the ceramic lining $[29,30,32]$.

Sub-analysis of marginal bone loss, complications and failures prosthesis were not performed, since the three studies that evaluated $8 \mathrm{~mm}$, only one [32] reported these variables.
Short implants are used in the posterior jaws due to their extensive bone resorption, since this region features greater mastication force [34]. Lekholm and Zarb [72] established a classification for organizing bone tissue into four categories with different degrees of density. Some authors suggested avoiding the use of short implants in areas with type IV bone found in the maxillary posterior, due to low density $[4,54]$. This generates masticatory overload, and increases failure rates compared to type II bone in the posterior mandible [14]. However, no differences were observed in relation to the survival rate and marginal bone loss for short implants in the analysis of specific jaws.

Thus, within all of the reported limitations, the results of the present review should be interpreted with caution due to the presence of uncontrolled confounding factors in the included studies [19], but the short implants must be used when necessary. 


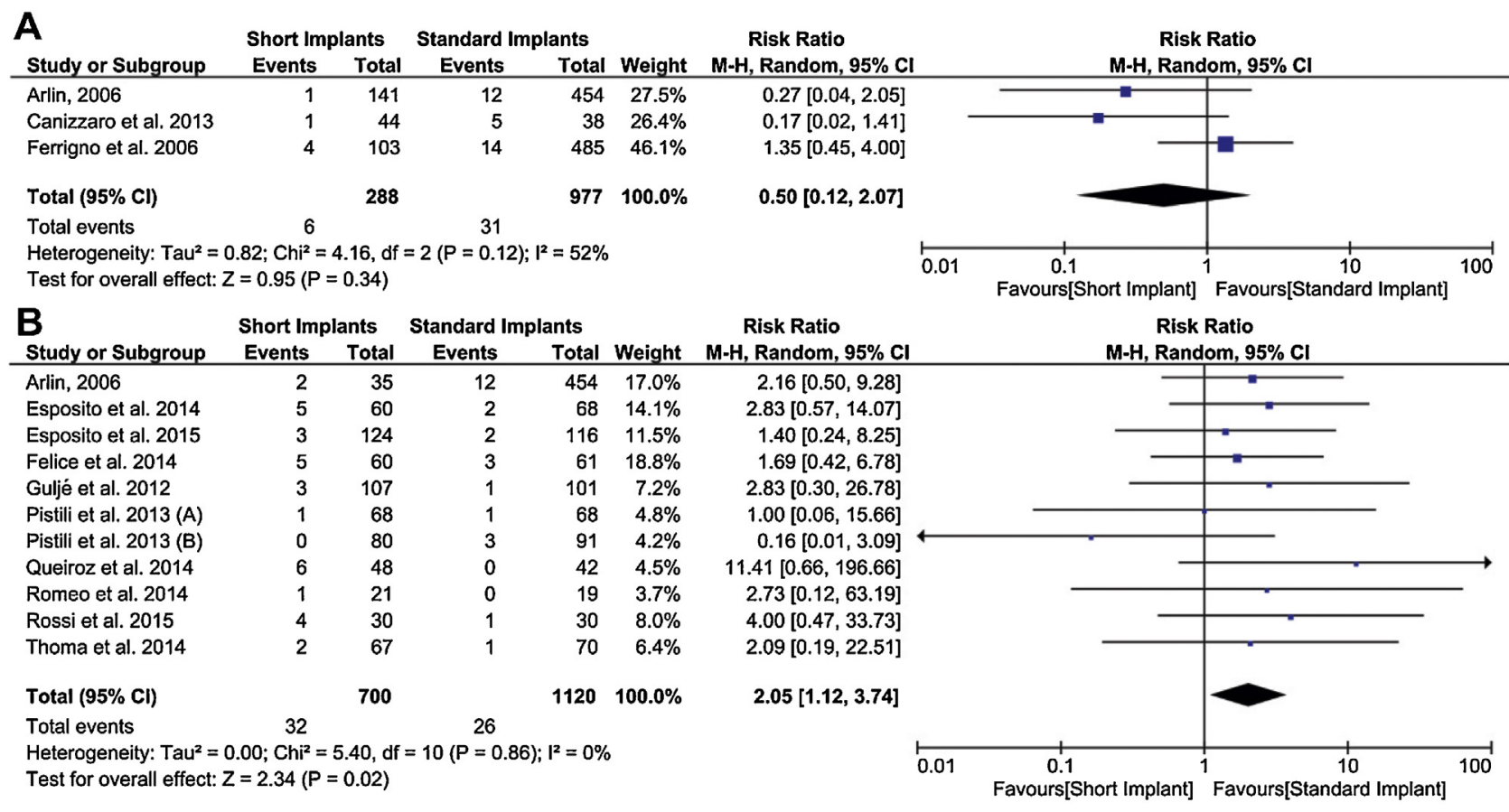

Fig. 5. Forest plot for the "short implants with $8 \mathrm{~mm}$ " (A), and "short implants less than $8 \mathrm{~mm}$ " (B) in the comparison between "standard implants" for survival rates of implants.

\begin{tabular}{|c|c|c|c|c|c|c|c|c|c|c|c|c|}
\hline $\mathbf{A}_{\text {Study or Subgroup }}$ & Shor & t Impla & & Stand & ard $\operatorname{lm}$ & plant & & Mean Difference & & Mean Dif & ifference & \\
\hline Canizzaro et al. 2013 & 0.41 & 0.42 & 44 & 0.72 & 0.41 & $3 \varepsilon$ & $\begin{array}{ll}8 & 11.4 \% \\
\end{array}$ & $-0.31[-0.49,-0.13]$ & & $=$ & & \\
\hline Esposito et al. 2014 & 1.58 & 0.54 & 60 & 1.89 & 0.57 & 68 & $8 \quad 11.2 \%$ & $-0.31[-0.50,-0.12]$ & & & & \\
\hline Esposito et al. 2015 & 0.39 & 0.3 & 124 & 0.43 & 0.25 & $11 \epsilon$ & $\begin{array}{ll}6 & 12.3 \%\end{array}$ & $-0.04[-0.11,0.03]$ & & & & \\
\hline Felice et al. 2014 & 2.24 & 0.47 & 60 & 3.01 & 0.74 & 61 & $1 \quad 10.9 \%$ & $-0.77[-0.99,-0.55]$ & & & & \\
\hline Guljé et al. 2012 & 0.06 & 0.6 & 107 & 0.02 & 0.6 & 10 & $1 \quad 11.5 \%$ & $0.04[-0.12,0.20]$ & & & & \\
\hline Pistili et al. 2013 (A) & 1.01 & 0.1 & 68 & 1.31 & 0.17 & 68 & $812.4 \%$ & $-0.30[-0.35,-0.25]$ & & $=$ & & \\
\hline Pistili et al. 2013 (B) & 1.21 & 0.19 & 80 & 0.99 & 0.06 & 91 & $1 \quad 12.4 \%$ & $0.22[0.18,0.26]$ & & & $=$ & \\
\hline Romeo et al. 2014 & 2.97 & 0.47 & 21 & 2.99 & 0.9 & 15 & $7.8 \%$ & $-0.02[-0.47,0.43]$ & & & & \\
\hline Rossi et al. 2015 & 2.3 & 0.52 & 30 & 2.64 & 0.56 & 30 & $0 \quad 10.2 \%$ & $-0.34[-0.61,-0.07]$ & & & & \\
\hline Total $(95 \% \mathrm{Cl})$ & & & 594 & & & 592 & $2 \quad 100.0 \%$ & $-0.20[-0.41,0.00]$ & & & & \\
\hline $\begin{array}{l}\text { Heterogeneity: } \mathrm{Tau}^{2}=0 \\
\text { Test for overall effect: } Z\end{array}$ & $\begin{array}{l}.09 ; \mathrm{Chi}^{2} \\
=1.91\end{array}$ & $\begin{array}{l}2=319 \\
P=0.0\end{array}$ & .35, df & $=8(\mathrm{P}$ & 0.0000 & $01) ;\left.\right|^{2}=$ & $=97 \%$ & & -2 & $\begin{array}{c}-1 \\
\text { Favours[Short Implant] }\end{array}$ & Favours[Standard Implant] & 2 \\
\hline B & Expe & rimen & & & ontrol & & & Mean Difference & & Mean Diff & fference & \\
\hline Study or Subgroup & Mean & SD & Total & Mean & SD & Total & Weight & IV, Random, $95 \% \mathrm{CI}$ & & IV, Randon & $\mathrm{m}, 95 \% \mathrm{Cl}$ & \\
\hline Canizzaro et al. 2013 & 0.41 & 0.42 & 38 & 0.72 & 0.41 & 44 & $23.7 \%$ & $-0.31[-0.49,-0.13]$ & & & & \\
\hline Esposito et al. 2014 & 1.36 & 0.57 & 34 & 1.74 & 0.57 & 38 & $20.1 \%$ & $-0.38[-0.64,-0.12]$ & & & & \\
\hline Pistili et al. 2013 (A) & 0.87 & 0.07 & 36 & 1.09 & 0.05 & 37 & $28.1 \%$ & $-0.22[-0.25,-0.19]$ & & $=$ & & \\
\hline Pistili et al. 2013 (B) & 1.02 & 0.06 & 39 & 0.94 & 0.05 & 44 & $28.1 \%$ & $0.08[0.06,0.10]$ & & & $=$ & \\
\hline Total $(95 \% \mathrm{Cl})$ & & & 147 & & & 163 & $100.0 \%$ & $-0.19[-0.41,0.03]$ & & & & \\
\hline $\begin{array}{l}\text { Heterogeneity: } \text { Tau }^{2}= \\
\text { Test for overall effect: }\end{array}$ & $\begin{array}{l}.04 ; \mathrm{Chi} \\
=1.68\end{array}$ & $\begin{array}{l}l^{2}=269 \\
(P=0 . C\end{array}$ & $\begin{array}{l}.21, \mathrm{df} \\
\text { 09) }\end{array}$ & $=3(\mathrm{P}$ & $<0.000$ & 01); $1^{2}$ & $=99 \%$ & & -1 & $\begin{array}{cc}-0.5 & 0 \\
\text { Favours[Short Implant] }\end{array}$ & $\begin{array}{c}0.5 \\
\text { Favours[Standard Implant] }\end{array}$ & 1 \\
\hline $\begin{array}{l}\text { C } \\
\text { Study or Subgroup }\end{array}$ & $\begin{array}{l}\text { Expe } \\
\text { Mean }\end{array}$ & $\begin{array}{l}\text { riment } \\
\text { SD }\end{array}$ & $\begin{array}{l}\text { al } \\
\text { Total }\end{array}$ & $\begin{array}{c}\text { Co } \\
\text { Mean }\end{array}$ & $\begin{array}{c}\text { ontrol } \\
\text { SD }\end{array}$ & Total & Weight & $\begin{array}{l}\text { Mean Difference } \\
\text { IV, Random, } 95 \% \mathrm{CI}\end{array}$ & & $\begin{array}{l}\text { Mean Diff } \\
\text { IV, Randon }\end{array}$ & $\begin{array}{l}\text { fference } \\
\mathrm{m}, 95 \% \mathrm{Cl}\end{array}$ & \\
\hline Esposito et al. 2014 & 1.79 & 0.51 & 26 & 1.97 & 0.57 & 30 & $24.1 \%$ & $-0.18[-0.46,0.10]$ & & & & \\
\hline Felice et al. 2014 & 2.24 & 0.47 & 60 & 3.01 & 0.74 & 61 & $24.7 \%$ & $-0.77[-0.99,-0.55]$ & & & & \\
\hline Pistili et al. 2013 (A) & 1.15 & 0.12 & 32 & 1.53 & 0.29 & 31 & $25.6 \%$ & $-0.38[-0.49,-0.27]$ & & 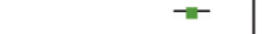 & & \\
\hline Pistili et al. 2013 (B) & 1.41 & 0.31 & 41 & 1.03 & 0.07 & 47 & $25.6 \%$ & $0.38[0.28,0.48]$ & & & - & \\
\hline Total $(95 \% \mathrm{Cl})$ & & & 159 & & & 169 & $100.0 \%$ & $-0.23[-0.76,0.30]$ & & & & \\
\hline $\begin{array}{l}\text { Heterogeneity: } \operatorname{Tau}^{2}= \\
\text { Test for overall effect: }\end{array}$ & $\begin{array}{l}.28 ; \mathrm{Ch} \\
=0.87\end{array}$ & $\begin{array}{l}i^{2}=15 \\
(P=0 .\end{array}$ & $\begin{array}{l}1.64, \mathrm{df} \\
39)\end{array}$ & $=3\langle P$ & $<0.000$ & $001) ;\left.\right|^{2}$ & $=98 \%$ & & -2 & $\begin{array}{cc}-1 & 1 \\
\text { Favours[Short Implant] } & 0\end{array}$ & Favours[Standard Implant] & 2 \\
\hline
\end{tabular}

Fig. 6. Forest plot for the "short implants" event in the comparison between "standard implants" for marginal bone loss (A), and individualized analyses of maxilla (B) and mandible (C). 


\begin{tabular}{|c|c|c|c|c|c|c|c|c|c|c|}
\hline $\begin{array}{l}\text { A } \\
\text { Study or Subgroup }\end{array}$ & \multicolumn{2}{|c|}{$\begin{array}{l}\text { Short Implants } \\
\text { Events Total }\end{array}$} & \multicolumn{2}{|c|}{ Standard Implants } & \multirow{2}{*}{ Weight } & $\begin{array}{l}\text { Risk Ratio } \\
\text { M-H, Random, } 95 \% \text { Cl }\end{array}$ & & \multicolumn{3}{|c|}{$\begin{array}{c}\text { Risk Ratio } \\
\mathrm{M}-\mathrm{H}, \text { Random, } 95 \% \mathrm{Cl}\end{array}$} \\
\hline Canizzaro et al. 2013 & 4 & 44 & 8 & 38 & & $0.43[0.14,1.32]$ & & & $T_{1}$ & \\
\hline Esposito et al. 2014 & 13 & 60 & 13 & 68 & $19.8 \%$ & $1.13[0.57,2.25]$ & & & & \\
\hline Esposito et al. 2015 & 3 & 116 & 2 & 124 & $9.5 \%$ & $1.60[0.27,9.42]$ & & & & \\
\hline Felice et al. 2014 & 6 & 60 & 25 & 61 & $18.3 \%$ & $0.24[0.11,0.55]$ & & & & \\
\hline Pistili et al. 2013 (A) & 8 & 68 & 22 & 68 & $19.2 \%$ & $0.36[0.17,0.76]$ & & & & \\
\hline Pistili et al. 2013 (B) & 0 & 80 & 14 & 91 & $4.9 \%$ & $0.04[0.00,0.65]$ & & & & \\
\hline Romeo et al. 2014 & 5 & 21 & 3 & 19 & $13.3 \%$ & $1.51[0.42,5.48]$ & & & & \\
\hline Total $(95 \% \mathrm{Cl})$ & & 449 & & 469 & $100.0 \%$ & $0.54[0.27,1.09]$ & & & & \\
\hline Total events & 39 & & 87 & & & & & & & \\
\hline $\begin{array}{l}\text { Heterogeneity: } \operatorname{Tau}^{2}= \\
\text { Test for overall effect: }\end{array}$ & $\begin{array}{l}.51 ; \text { Chì }^{2}= \\
=1.73(P=\end{array}$ & $\begin{array}{l}17.42, \mathrm{df} \\
0.08)\end{array}$ & $f=6(P=0.0$ & $3) ; 1^{2}=66$ & & & 0.01 & $\begin{array}{c}0.1 \\
\text { Favours[Short Implant] }\end{array}$ & $\begin{array}{cc} & 10 \\
\text { Favours[Standard Implant] }\end{array}$ & 100 \\
\hline $\begin{array}{l}\text { B } \\
\text { Study or Subgroup }\end{array}$ & $\begin{array}{l}\text { Short Imp| } \\
\text { Events }\end{array}$ & $\begin{array}{l}\text { ants } \\
\text { Total }\end{array}$ & $\begin{array}{l}\text { Standard In } \\
\text { Events }\end{array}$ & $\begin{array}{l}\text { lants } \\
\text { Total }\end{array}$ & Weight & $\begin{array}{c}\text { Risk Ratio } \\
\text { M-H, Random, } 95 \% \mathrm{Cl}\end{array}$ & & $\begin{array}{r}\text { Risk F } \\
\text { M-H, Rando }\end{array}$ & $\begin{array}{l}\text { Ratio } \\
\text { om, } 95 \% \mathrm{Cl}\end{array}$ & \\
\hline Canizzaro et al. 2013 & 1 & 44 & 2 & 38 & $10.8 \%$ & $0.43[0.04,4.58]$ & & & & \\
\hline Esposito et al. 2014 & 3 & 60 & 0 & 68 & $7.0 \%$ & $7.92[0.42,150.24]$ & & & & \\
\hline Esposito et al. 2015 & 2 & 116 & 2 & 124 & $16.0 \%$ & $1.07[0.15,7.47]$ & & & & \\
\hline Felice et al. 2014 & 5 & 60 & 5 & 61 & $42.8 \%$ & $1.02[0.31,3.33]$ & & & & \\
\hline Pistili et al. 2013 (A) & 1 & 68 & 2 & 68 & $10.7 \%$ & $0.50[0.05,5.39]$ & & & & \\
\hline Pistili et al. 2013 (B) & 0 & 80 & 2 & 91 & $6.6 \%$ & $0.23[0.01,4.66]$ & & & & \\
\hline Romeo et al. 2014 & 1 & 21 & 0 & 19 & $6.1 \%$ & $2.73[0.12,63.19]$ & & & & \\
\hline Total $(95 \% \mathrm{Cl})$ & & 449 & & 469 & $100.0 \%$ & $0.96[0.44,2.09]$ & & & & \\
\hline Total events & 13 & & 13 & & & & & & & \\
\hline $\begin{array}{l}\text { Heterogeneity: } \operatorname{Tau}^{2}=0 \\
\text { Test for overall effect: } Z\end{array}$ & $\begin{array}{l}00 ; \mathrm{Chi}^{2}=4 \\
=0.10(\mathrm{P}=\end{array}$ & $\begin{array}{l}.05, \mathrm{df}= \\
0.92)\end{array}$ & $=6\langle P=0.67$ & & & & 0.01 & $\begin{array}{c}1 \\
0.1 \\
\text { Favours[Sh }\end{array}$ & $\begin{array}{c}10 \\
\text { Favours[Standard Implant] }\end{array}$ & $100^{\circ}$ \\
\hline
\end{tabular}

Fig. 7. Forest plot for the "short implants" event in the comparison between "standard implants" for complication rates (A), and prosthesis failures (B).

Cases include low vertical bone height and patients for whom complementary surgical procedures are not favorable.

\section{Conclusion}

Short implants showed marginal bone loss, prosthesis failures and complication rates similar to standard implants, being considered a predictable treatment for posterior jaws, especially in cases that require complementary surgical procedures. However, short implants with length less than $8 \mathrm{~mm}(4-7 \mathrm{~mm})$ should be used with caution because they present greater risks for implant failures when compared to standard implants.

\section{References}

[1] J. Pennington, S. Parker, Improving quality of life using removable and fixed implant prostheses, Compend. Contin. Educ. Dent. 33 (2012) 268-276.

[2] L. Feller, R. Chandran, R.A. Khammissa, R. Meyerov, Y. Jadwat, M. Bouckaert, et al., Osseointegration: biological events in relation to characteristics of the implant surface, South Afr. Dent. Assoc. 69 (2014) 112-117.

[3] A.A. Al-Hashedi, T.B. Taiyeb Ali, N. Yunus, Short Dental Implants: An Emerging Concept in Implant Treatment, 45, Quintessence International, 2014, pp. 499514.

[4] F. Renouard, D. Nisand, Impact of implant length and diameter on survival rates, Clin. Oral Implants Res. 17 (Suppl 2) (2006) 35-51.

[5] M. Esposito, M.G. Grusovin, P. Felice, G. Karatzopoulos, H.V. Worthington, P. Coulthard, Interventions for replacing missing teeth: horizontal and vertical bone augmentation techniques for dental implant treatment, Cochrane Database Syst. Rev. 7 (2009) Cd003607.

[6] T.P. Queiroz, S.C. Aguiar, R. Margonar, A.P. de Souza Faloni, R. Gruber, E.R. Luvizuto, Clinical study on survival rate of short implants placed in the posterior mandibular region: resonance frequency analysis, Clin. Oral Implants Res. 26 (2015) 1036-1042.

[7] M. Esposito, P. Felice, H.V. Worthington, Interventions for replacing missing teeth: augmentation procedures of the maxillary sinus, Cochrane Database Syst. Rev. 5 (2014) Cd008397.

[8] B.S. Sotto-Maior, P.M. Senna, J.P. Silva-Neto, M.A. de Arruda Nobilo, A.A. Cury, Influence of crown-to-implant ratio on stress around single short-wide implants: a photoelastic stress analysis, J. Prosthodont. 24 (2015) 52-56.

[9] G. Telleman, G.M. Raghoebar, A. Vissink, L. den Hartog, J.J. Huddleston Slater, H. J. Meijer, A systematic review of the prognosis of short $(<10 \mathrm{~mm})$ dental implants placed in the partially edentulous patient, J. Clin. Periodontol. 38 (2011) 667-676.

[10] L.A. Mezzomo, R. Miller, D. Triches, F. Alonso, R.S. Shinkai, Meta-analysis of single crowns supported by short $(<10 \mathrm{~mm})$ implants in the posterior region, $\mathrm{J}$. Clin. Periodontol. 41 (2014) 191-213.
[11] S.A. Lee, C.T. Lee, M.M. Fu, W. Elmisalati, S.K. Chuang, Systematic review and meta-analysis of randomized controlled trials for the management of limited vertical height in the posterior region: short implants ( 5 to $8 \mathrm{~mm}$ ) vs longer implants $(>8 \mathrm{~mm})$ in vertically augmented sites, Int. J. Oral Maxillofac. Implants 29 (2014) 1085-1097.

[12] E. Anitua, M.H. Alkhraist, L. Pinas, L. Begona, G. Orive, Implant survival and crestal bone loss around extra-short implants supporting a fixed denture: the effect of crown height space, crown-to-implant ratio, and offset placement of the prosthesis, Int. J. Oral Maxillofac. Implants 29 (2014) 682-689.

[13] A. Quaranta, M. Piemontese, G. Rappelli, G. Sammartino, M. Procaccini, Technical and biological complications related to crown to implant ratio: a systematic review, Implant Dent. 23 (2014) 180-187.

[14] M.C. Goiato, D.M. dos Santos, J.F. Santiago Jr., A. Moreno, E.P. Pellizzer Longevity of dental implants in type IV bone: a systematic review, Int. J. Oral Maxillofac. Surg. 43 (2014) 1108-1116.

[15] J.A. Mendonca, C.E. Francischone, P.M. Senna, A.E. Matos de Oliveira, B.S. SottoMaior, A retrospective evaluation of the survival rates of splinted and nonsplinted short dental implants in posterior partially edentulous jaws, J. Periodontol. 85 (2014) 787-794.

[16] C.E. Misch, J. Steignga, E. Barboza, F. Misch-Dietsh, L.J. Cianciola, C. Kazor, Short dental implants in posterior partial edentulism: a multicenter retrospective 6 year case series study, J. Periodontol. 77 (2006) 1340-1347.

[17] E. Anitua, M.H. Alkhraisat, G. Orive, Novel technique for the treatment of the severely atrophied posterior mandible, Int. J. Oral Maxillofac. Implants 28 (2013) 1338-1346.

[18] F. Rossi, N.P. Lang, E. Ricci, L. Ferraioli, C. Marchetti, D. Botticelli, Early loading of 6 -mm-short implants with a moderately rough surface supporting single crowns-a prospective 5-year cohort study, Clin. Oral Implants Res. 26 (2015) 471-477.

[19] C. Slotte, A. Gronningsaeter, A.M. Halmoy, L.O. Ohrnell, A. Mordenfeld, S. Isaksson, et al., Four-millimeter-long posterior-mandible implants: 5-year outcomes of a prospective multicenter study, Clin. Implant Dent. Relat. Res. (2014), doi:http://dx.doi.org/10.1111/cid.12252.

[20] D. Moher, A. Liberati, J. Tetzlaff, D.G. Altman, Preferred reporting items for systematic reviews and meta-analyses: the PRISMA statement, Int. J. Surg. 8 (2010) 336-341.

[21] M. Schroeder, A. Reis, I. Luque-Martinez, A.D. Loguercio, D. Masterson, L.C. Maia, Effect of enamel bevel on retention of cervical composite resin restorations: a systematic review and meta-analysis, J. Dent. 43 (2015) 777 788.

[22] B.R. Chrcanovic, T. Albrektsson, A. Wennerberg, Dental implants inserted in fresh extraction sockets versus healed sites: a systematic review and metaanalysis, J. Dent. 43 (2015) 16-41.

[23] L.F. Lopes, V.F. da Silva, J.F. Santiago Jr., S.R. Panzarini, E.P. Pellizzer, Placement of dental implants in the maxillary tuberosity: a systematic review, Int. J. Oral Maxillofac. Surge. 44 (2015) 229-238.

[24] A.R. Jadad, R.A. Moore, D. Carroll, C. Jenkinson, D.J. Reynolds, D.J. Gavaghan, et al., Assessing the quality of reports of randomized clinical trials: is blinding necessary? Controll. Clin. Trials 17 (1996) 1-12.

[25] M. Esposito, C. Barausse, R. Pistilli, V. Checchi, M. Diazzi, M.R. Gatto, et al., Posterior jaws rehabilitated with partial prostheses supported by $4.0 \times 4.0 \mathrm{~mm}$ 
or by longer implants: four-month post-loading data from a randomised controlled trial, Eur. J. Oral Implantol. 8 (2015) 221-230.

[26] F. Rossi, D. Botticelli, G. Cesaretti, E. De Santis, S. Storelli, N.P. Lang, Use of short implants $(6 \mathrm{~mm})$ in a single-tooth replacement: a 5-year follow-up prospective randomized controlled multicenter clinical study, Clin. Oral Implants Res. (2015), doi:http://dx.doi.org/10.1111/clr.12564 [Epub ahead of print].

[27] D.S. Thoma, R. Haas, M. Tutak, A. Garcia, G.P. Schincaglia, C.H. Hammerle, Randomized controlled multicentre study comparing short dental implants $(6 \mathrm{~mm})$ versus longer dental implants $(11-15 \mathrm{~mm})$ in combination with sinus floor elevation procedures. Part 1: demographics and patient-reported outcomes at 1 year of loading, J. Clin. Periodontol. 42 (2015) 72-80.

[28] M. Esposito, R. Pistilli, C. Barausse, P. Felice, Three-year results from a randomised controlled trial comparing prostheses supported by $5-\mathrm{mm}$ long implants or by longer implants in augmented bone in posterior atrophic edentulous jaws, Eur. J. Oral Implantol. 7 (2014) 383-395.

[29] P. Felice, G. Cannizzaro, C. Barausse, R. Pistilli, M. Esposito, Short implants versus longer implants in vertically augmented posterior mandibles: a randomised controlled trial with 5-year after loading follow-up, Eur. J. Oral Implantol. 7 (2014) 359-369.

[30] E. Romeo, S. Storelli, G. Casano, M. Scanferla, D. Botticelli, Six-mm versus 10 mm long implants in the rehabilitation of posterior edentulous jaws: a 5-year follow-up of a randomised controlled trial, Eur. J. Oral Implantol. 7 (2014) 371 381.

[31] R. Pistilli, P. Felice, M. Piattelli, M. Gessaroli, E. Soardi, C. Barausse, et al., Posterior atrophic jaws rehabilitated with prostheses supported by $5 \times 5 \mathrm{~mm}$ implants with a novel nanostructured calcium-incorporated titanium surface or by longer implants in augmented bone. One-year results from a randomised controlled trial, Eur. J. Oral Implantol. 6 (2013) 343-357.

[32] G. Cannizzaro, P. Felice, A.F. Minciarelli, M. Leone, P. Viola, M. Esposito, Early implant loading in the atrophic posterior maxilla: 1-stage lateral versus crestal sinus lift and $8 \mathrm{~mm}$ hydroxyapatite-coated implants: a 5-year randomised controlled trial, Eur. J. Oral Implantol. 6 (2013) 13-25.

[33] R. Pistilli, P. Felice, G. Cannizzaro, M. Piatelli, V. Corvino, C. Barausse, et al., Posterior atrophic jaws rehabilitated with prostheses supported by $6 \mathrm{~mm}$ long $4 \mathrm{~mm}$ wide implants or by longer implants in augmented bone: one-year postloading results from a pilot randomised controlled trial, Eur. J. Oral Implantol. 6 (2013) 359-372.

[34] F. Gulje, I. Abrahamsson, S. Chen, C. Stanford, H. Zadeh, R. Palmer, Implants of $6 \mathrm{~mm}$ vs: $11 \mathrm{~mm}$ lengths in the posterior maxilla and mandible: a 1-year multicenter randomized controlled trial, Clin. Oral Implants Res. 24 (2013) 1325-1331.

[35] M.L. Arlin, Short dental implants as a treatment option: results from an observational study in a single private practice, Int. J. Oral Maxillofac. Implants 21 (2006) 769-776.

[36] N. Ferrigno, M. Laureti, S. Fanali, Dental implants placement in conjunction with osteotome sinus floor elevation: a 12-year life-table analysis from a prospective study on 588 ITI implants, Clin. Oral Implants Res. 17 (2006) 194-205.

[37] G. Cannizzaro, M. Leone, C. Torchio, P. Viola, M. Esposito, Immediate versus early loading of 7-mm-long flapless-placed single implants: a split-mouth randomised controlled clinical trial, Eur. J. Oral Implantol. 1 (2008) 277-292.

[38] G. Cannizzaro, P. Felice, M. Leone, P. Viola, M. Esposito, Early loading of implants in the atrophic posterior maxilla: lateral sinus lift with autogenous bone and Bio-Oss versus crestal mini sinus lift and 8-mm hydroxyapatitecoated implants. A randomised controlled clinical trial, Eur. J. Oral Implantol. 2 (2009) 25-38.

[39] J. D'Haese, T. Van De Velde, L. Elaut, H. De Bruyn, A prospective study on the accuracy of mucosally supported stereolithographic surgical guides in fully edentulous maxillae, Clin. Implant Dent. Relat. Res. 14 (2012) 293-303.

[40] M. Esposito, G. Pellegrino, R. Pistilli, P. Felice, Rehabilitation of postrior atrophic edentulous jaws: prostheses supported by $5 \mathrm{~mm}$ short implants or by longer implants in augmented bone? One-year results from a pilot randomised clinical trial, Eur. J. Oral Implantol. 4 (2011) 21-30.

[41] M. Esposito, G. Cannizzaro, E. Soardi, R. Pistilli, M. Piattelli, V. Corvino, et al, Posterior atrophic jaws rehabilitated with prostheses supported by $6 \mathrm{~mm}-$ long, $4 \mathrm{~mm}$-wide implants or by longer implants in augmented bone: preliminary results from a pilot randomised controlled trial, Eur. J. Oral Implantol. 5 (2012) 19-33.

[42] P. Felice, V. Checchi, R. Pistilli, A. Scarano, G. Pellegrino, M. Esposito, Bone augmentation versus $5-\mathrm{mm}$ dental implants in posterior atrophic jaws: fourmonth post-loading results from a randomised controlled clinical trial, Eur. J. Oral Implantol. 2 (2009) 267-281.

[43] P. Felice, G. Cannizzaro, V. Checchi, C. Marchetti, G. Pellegrino, P. Censi, et al., Vertical bone augmentation versus 7-mm-long implants in posterior atrophic mandibles: results of a randomised controlled clinical trial of up to 4 months after loading, Eur. J. Oral Implantol. 2 (2009) 7-20.

[44] P. Felice, G. Pellegrino, L. Checchi, R. Pistilli, M. Esposito, Vertical augmentation with interpositional blocks of anorganic bovine bone vs: 7-mm-long implants in posterior mandibles: 1-year results of a randomized clinical trial, Clin. Oral Implants Res. 21 (2010) 1394-1403.

[45] P. Felice, R. Pistilli, M. Piattelli, E. Soardi, V. Corvino, M. Esposito, Posterior atrophic jaws rehabilitated with prostheses supported by $5 \times 5 \mathrm{~mm}$ implants with a novel nanostructured calcium-incorporated titanium surface or by longer implants in augmented bone. Preliminary results from a randomised controlled trial, Eur. J. Oral Implantol. 5 (2012) 149-161.
[46] F.L. Guljé, G.M. Raghoebar, A. Vissink, H.J. Meijer, Single restorations in the resorbed posterior mandible supported by 6-mm implants: a 1-year prospective case series study, Clin. Implant Dent. Relat. Res. 2 (Suppl. 2) (2015) e465-e471, doi:http://dx.doi.org/10.1111/cid.12272 Epub 2014 Sep 5.

[47] D. Penarrocha-Oltra, A. Aloy-Prosper, J. Cervera-Ballester, M. PenarrochaDiago, L. Canullo, Implant treatment in atrophic posterior mandibles: vertical regeneration with block bone grafts versus implants with $5.5-\mathrm{mm}$ intrabony length, Int. J. Oral Maxillofac. Implants 29 (2014) 659-666.

[48] F. Pieri, N.N. Aldini, M. Fini, C. Marchetti, G. Corinaldesi, Preliminary 2-year report on treatment outcomes for 6-mm-long implants in posterior atrophic mandibles, Int. J. Prosthodont. 25 (2012) 279-289.

[49] M.A. Sanchez-Garces, X. Costa-Berenguer, C. Gay-Escoda, Short implants: a descriptive study of 273 implants, Clin. Implant Dent. Relat. Res. 14 (4) (2012) 508-516.

[50] R. Nedir, N. Nurdin, P. Khoury, M. Bischof, Short implants placed with or without grafting in atrophic sinuses: The 3-year results of a prospective randomized controlled study, Clin. Implant Dent. Relat. Res. (2015), doi:http:// dx.doi.org/10.1111/cid.12279.

[51] P. Felice, E. Soardi, G. Pellegrino, R. Pistilli, C. Marchetti, M. Gessaroli, et al., Treatment of the atrophic edentulous maxilla: short implants versus bone augmentation for placing longer implants: five-month post-loading results of a pilot randomised controlled trial, Eur. J. Oral Implantol. 4 (2011) 191-202.

[52] M. Esposito, C. Barausse, R. Pistilli, G. Sammartino, G. Grandi, P. Felice, Short implants versus bone augmentation for placing longer implants in atrophic maxillae: one-year post-loading results of a pilot randomised controlled trial, Eur. J. Oral Implantol. 8 (2015) 257-268.

[53] J.R. Landis, G.G. Koch, The measurement of observer agreement for categorical data, Biometrics 33 (1977) 159-174.

[54] D. Nisand, F. Renouard, Short implant in limited bone volume, Periodontology 200066 (2014) 72-96.

[55] G. Tawil, N. Aboujaoude, R. Younan, Influence of prosthetic parameters on the survival and complication rates of short implants, Int. J. Oral Maxillofac. Implants 21 (2006) 275-282.

[56] R. Gapski, H.L. Wang, P. Mascarenhas, N.P. Lang, Critical review of immediate implant loading, Clin. Oral Implants Res. 14 (2003) 515-527.

[57] S. Annibali, M.P. Cristalli, D. Dell'Aquila, I. Bignozzi, G. La Monaca, A. Pilloni, Short dental implants: a systematic review, J. Dent. Res. 91 (2012) 25-32.

[58] L. Pierrisnard, F. Renouard, P. Renault, M. Barquins, Influence of implant length and bicortical anchorage on implant stress distribution, Clin. Implant Dent. Relat. Res. 5 (2003) 254-262.

[59] B. Pommer, S. Frantal, J. Willer, M. Posch, G. Watzek, G. Tepper, Impact of dental implant length on early failure rates: a meta-analysis of observational studies, J. Clin. Periodontol. 38 (2011) 856-863.

[60] S. Kotsovilis, I. Fourmousis, I.K. Karoussis, C. Bamia, A systematic review and meta-analysis on the effect of implant length on the survival of rough-surface dental implants, J. Periodontol. 80 (2009) 1700-1718.

[61] F. Renouard, D. Nisand, Short implants in the severely resorbed maxilla: a 2-year retrospective clinical study, Clin. Implant Dent. Relat. Res. 7 (2005) 104-110.

[62] D. Deporter, Short dental implants: what works and what doesn't? A literature interpretation, Int. J. Periodont. Restor. Dent. 33 (2013) 457-464.

[63] F. Ramos Verri, J.F. Santiago Junior, D.A. de Faria Almeida, G.B. de Oliveira, V.E. de Souza Batista, H. Marques Honório, et al., Biomechanical influence of crown-to-implant ratio on stress distribution over internal hexagon short implant: 3-D finite element analysis with statistical test, J. Biomech. 48 (2015) $138-145$.

[64] S.P. Sun, I.S. Moon, K.H. Park, D.W. Lee, Effect of crown to implant ratio and anatomical crown length on clinical conditions in a single implant: retrospective cohort study, Clin. Implant Dent. Relat. Res. 17 (2015) 724-731.

[65] E.P. Pellizzer, C.C. de Mello, J.F. Santiago Junior, V.E. de Souza Batista, D.A. de Faria Almeida, F.R. Verri, Analysis of the biomechanical behavior of short implants: the photo-elasticity method, Mater. Sci. Eng. C Mater. Biol. Appl. 55 (2015) 187-192.

[66] I. Ortega-Oller, F. Suarez, P. Galindo-Moreno, L. Torrecillas-Martinez, A. Monje, A. Catena, et al., The influence of implant diameter on its survival: a metaanalysis based on prospective clinical trials, J. Periodontol. 85 (2014) 569-580.

[67] M. Coelho Goiato, A.A. Pesqueira, D.M. Santos, M.F. Haddad, A. Moreno, Photoelastic stress analysis in prosthetic implants of different diameters: mini, narrow, standard or wide, J. Clin. Diagn. Res. 8 (2014) 86-90.

[68] F. Javed, G.E. Romanos, Role of implant diameter on long-term survival of dental implants placed in posterior maxilla: a systematic review, Clin. Oral Invest. 19 (2015) 1-10.

[69] T. Albrektsson, G. Zarb, P. Worthington, A.R. Eriksson, The long-term efficacy of currently used dental implants: a review and proposed criteria of success, Int. J. Oral Maxillofac. Implants 1 (1986) 11-25.

[70] S.A. Lee, C.T. Lee, M.M. Fu, W. Elmisalati, S.K. Chuang, Systematic review and meta-analysis of randomized controlled trials for the management of limited vertical height in the posterior region: short implants (5 to $8 \mathrm{~mm}$ ) vs longer implants $(>8 \mathrm{~mm})$ in vertically augmented sites, Int. J. Oral Maxillofac. Implants 29 (2014) 1085-1097.

[71] D.S. Thoma, M. Zeltner, J. Hüsler, C.H. Hämmerle, R.E. Jung, EAO supplement working group 4-EAO CC 2015 short implants versus sinus lifting with longer implants to restore the posterior maxilla: a systematic review, Clin. Oral Implants Res. 11 (2015) 154-169.

[72] U. Lekholm, G. Zarb, Patient selection and preparation, in: P.I. Brånemark, G. Zarb, T. Albrektsson (Eds.), Tissue-integrated prostheses, Quintessence, Chicago, 1985, pp. 199--211. 